\title{
Herbal remedies used for the management of urolithiasis in Abbottabad, Northern Pakistan
}

\author{
Ghulam Mujtaba Shah ${ }^{1 a}$, Nasir Shad ${ }^{2 a}$, Muhammad Sajid $^{3}$, Asma $^{1}$, Abid Naeem ${ }^{*}$, Fahim Ullah Khan ${ }^{3}$, \\ Nadia Jabeen ${ }^{3}$, Mazna Urooj ${ }^{1}$, Said Muhammad ${ }^{1} \&$ Muhammad Hasnain $^{5}$ \\ ${ }^{1}$ Department of Botany, Hazara University Mansehra, Pakistan \\ ${ }^{2}$ Jiangxi Provincial Key Laboratory of Silviculture, College of Forestry, Jiangxi Agricultural University, Nanchang 330045, China \\ ${ }^{3}$ Department of Agricultural, Hazara University Mansehra, Pakistan \\ ${ }^{4}$ Key Laboratory of Modern Preparation of Traditional Chinese Medicine, Ministry of Education, Jiangxi University of Traditional Chinese \\ Medicine, 1688 Meiling Road, Nanchang 330006, PR China \\ ${ }^{5}$ State Environmental Protection Key Laboratory of Wetland Ecology and Vegetation Restoration, School of Environment, Northeast Normal \\ University, Changchun 130117, China \\ ${ }^{a}$ These authors contributed equally to this article \\ *Email: anaeemktk@gmail.com
}

\section{ARTICLE HISTORY}

Received: 02 May 2021

Accepted: 26 June 2021

Available online: 23 August 2021

\section{KEYWORDS}

Herbal medicine

Traditional knowledge

Ethnobotany

Urolithiasis

Kidney stone

\begin{abstract}
The current study was performed to evaluate the ethnobotanical uses of medicinal plants to treat and prevent kidney diseases, especially urolithiasis in the Abbottabad region, Northern Pakistan. Field surveys were conducted from 2014-2016 in various rural and tribally dwelled hilly areas like Ayubia National Park, Sherwan and Thandiani. Ethnobotanical information about the medicinal plants employed to treat urinary ailments was obtained from well-informed sources like local healers (hakims) and residents (men/women), who had vast knowledge of local plants uses. The questionnaire method was adopted to record the information and queries were made to verify the information. Informed consent was obtained from each informant before conducting the interview process. Quantitative ethnobotanical indices were calculated for each recorded species. Correlation analysis between the RFC, UV and FL\% was tested by Pearson's correlation, SPSS (ver. 16). A total of 38 plant species belonging to 27 families were being used in the study area to treat Urolithiasis or kidney stone diseases. Asteraceae was the most dominant family with 5 species. Local people used different methods of preparation for different plant parts; among them, decoction was the popular and dominant way of preparation (52.6\%), followed by powder (18.4\%), extracts (15.7\%), juice (7.8\%) and cooked (5.2\%). The highest UV was recorded for Rumex acetosa L. (1.14) followed by Agrimonia eupatoria L. (1.1), Equisetum ramosissimum Desf. (1.05), Aerva lanata L. (1.04), Eclipta alba Hassak. (1.03). Moreover, twenty-three species were recoded with above $50 \%$ Fidelity level. It was found that most of the plant species (16 species) were explicitly used to remove kidney stones. All the obtained data about the ethnomedicinal uses of plants to treat urinary tract ailments are alphabetically categorized to their botanical name/family, local name, phytoconstituents, dosage and route of administration, along with quantitative indices value. All the collected ethnomedicinal plants require a thorough scientific investigation for isolation, identification, biochemical assays, toxicities and evaluation of pharmacological activities of the phytoconstituents, especially of the plants recorded with a high-fidelity level before their usage in clinics.
\end{abstract}

\section{Introduction}

Urolithiasis or kidney stone formation is the third leading urinary system disease affecting $10-15 \%$ of the population worldwide. Generally, it emanates from tiny crystals, which later results in stone, and subsequently grows further and accumulates on the interior surfaces of the kidney (1). Kidney stones or calculi are named based on the identified locations in the urinary system, such as renal calculi, urinary tract stone disease, urinary calculi, urolithiasis, nephrolithiasis and ureterolithiasis. Urinary stones can be removed/expelled via urine if smaller in size through the ureter from the kidney (2). It is observed that sometimes the stones may not pass through the ureter, which produces severe pain if not managed properly, resulting in serious medical consequences 
like extreme obstruction, infection, haemorrhage and hydronephrosis in the urinary system.

The major health problems faced by the people of Pakistan are linked to the kidney, and recently more than 1 million cases of kidney stones were reported (3). It is estimated that $15 \%$ of all the people in Pakistan will suffer from urolithiasis at some point in life. Men tend to be affected more frequently than women (4). Moreover, urolithiasis is the most common illness amongst the people of Northern Pakistan. Different treatment options are available to remove calculi, such as surgical removal, lithotripsy and laparoscopy. Despite the higher treatment costs, these techniques may produce a risk of causing acute renal injury, which decreases renal function and also recurrence of calculi observed in some cases with a possible recurrence rate of $10 \%, 33 \%$ and $50 \%$ at 1 st, 5th and 10th year respectively (5). Costly diagnostic and treatment procedures for the therapeutic management of patients require better adequate alternative therapies. Phytochemicals are responsible for the therapeutic activity of plant species and can treat various ailments and possess potential antiinflammatory, antibacterial, analgesic, diuretic, antioxidant and antifungal properties. Natural products derived from medicinal plants, either as pure compounds or as extracts, provide opportunities for developing new drug leads due to the unmatched chemical diversity and lower cost compared to their synthetic chemical counter-parts (6). Due to the recent surge in demands for chemical diversity in screening programmes, search for therapeutic drugs of herbal origin increased due to their attractive properties worldwide. Botanicals and herbal preparations for medicinal purposes contain different types of bioactive compounds like flavonoids, alkaloids, saponins, tannins, sterols and other organic and inorganic constituents. Some medicinal plants and marketed herbal preparations are reported to be effective in treating and preventing kidney stones recurrence with fewer side effects (7). In recent times, the discovery of antilithiatic drugs from natural products has gained a lot of importance.

The practiced medicinal plants by traditional healers and locals for the cure of kidney stones possess various bioactive chemical compounds, and some of the chemical constituents from different plant species have been used in anti-urolithiatic formulations (6). Various phytoconstituents have been isolated from traditionally used medicinal plants and have been found to be effective in preventing kidney disease/stones $(6,8)$. Diet from natural food (vegetative plants and fruits) also plays a vital role in either preventing or promoting the formation of kidney stones, depending on the types of nutrients and chemical constituents found in the plants (6). Those plants which contain phytoconstituents like (flavonoids, alkaloids, tannins, saponins, phenolic compounds, organic and inorganic constituents, plant proteins etc.) are responsible for anti-urolithiatic activity (9). The World Health Organization (WHO) stated that almost 4 billion (80\%) people worldwide take advantage of herbal remedies for their healthcare needs. Furthermore, the remaining $25 \%$ of conventional drugs marketed in developed countries are mostly derived from natural products such as vinblastine, quinine, reserpine and aspirin (10). It is estimated that about 50000-70.000 plant species are utilized for medicinal purposes by traditional and modern healthcare systems worldwide (11). Pakistan has different climatic and ecological zones enriched with floral diversity, containing almost 6000 species of important flowering plants, including 500 species of extreme therapeutic importance. Some of these plants are used in the locally practiced medical system (Hikmat) (12).

A thorough literature search revealed that a lot of work had been done on the medicinal plants in the Abbottabad region (13-20). However, yet no report is available regarding kidney diseases. Therefore, it would be important to document these medicinal plants employed for treating kidney stones. The current study will not only be helpful in the conservation of resources but also helps in preserving the fast-disappearing traditional knowledge and may provide a base for pharmacological research.

\section{Materials and Methods}

This study was authorized by the Department of Botany and Office of Research, Innovation and Commercialization of Hazara University (ORIC-HU), Mansehra, Pakistan. Informed consent was obtained from each informant before conducting the interview process.

\section{Study area}

The Abbottabad district covers an area of $1967 \mathrm{~km}^{2}$ and falls between $34^{\circ} 9^{\prime}-34^{\circ} 21^{\prime}$ North latitude and $73^{\circ} 10^{\prime}-73^{\circ} 13^{\prime}$ East longitude, predominantly mountainous terrain (Fig. 1). The average peak elevation ranges from $2500-2700 \mathrm{~m}$ and the Miranjani peak $(3313 \mathrm{~m})$ is the highest point in the district. These peaks dominate the landscape and are a part of the lesser Himalayas. The Dongagali

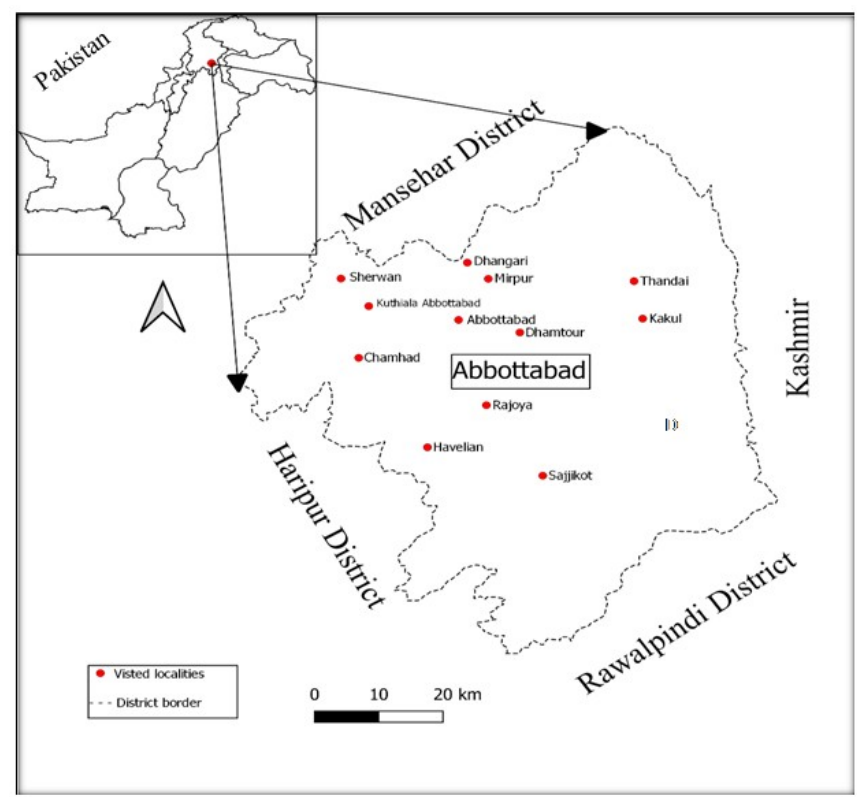

Fig. 1. Map of the study area (visited localities) Abbottabad District, Khyber Pakhtunkhwa, Pakistan. 
peaks unite with the right banks of the Jhelum and Kunhar river from the north. It includes various offshoots that differ in altitudes from 2500-2700 m. Several other peaks also project around this range, especially from the west, with 600-1500 $\mathrm{m}$ of range. The overall population of Abbottabad is around 1169432 and the urban population is estimated to be 1519751. The topography of Abbottabad is mainly composed of gullies and steep slopes, with metamorphic rocks formed of limestone. Recently, frequent deforestation and pasturage triggered soil erosion, which has made the parent rock shallower and silty loess (21). The average temperature in the region ranges from 34.3-3.4 ${ }^{\circ} \mathrm{C}$, with occasional snow. Most rainfall occurs in winter and monsoon; the average being recorded is around $1200 \mathrm{~mm}$ per year. The major tribes of the region include Gujjar, Awan, Karlal, Syed, Jadoon, Abbasi, Rajput, Kashmiri and Mughal. Most of the population depends on forests and agriculture (primary occupation), although cattle grazing occurs near the adjacent hilly areas. Nevertheless, some people are also involved in local labour and trade. The most commonly grown crops in the area include rice, maize, wheat, tobacco etc. Recently, due to increased illegal tree cuttings, extensive fires, illegal settlements, urban encroachment and growing pollution, the area is under severe threat (22).

\section{Ethnobotanical data collection}

Ethnobotanical data were collected following ethical guidelines with informed consent. Questionnaires and semi-structured interviews (SSI) were conducted in spring and summer (2014-2016) at 15 different localities in the Lesser Himalayas. About 95 key informants (15-20 per site and aged 25 to 75 years) with reliable and in-depth knowledge of traditionally used medicinal plants against urolithiasis and residing there for (30 years) were interviewed several times regarding the healing properties against urolithiasis (Table 1). Questions asked to the informants about the medicinally used plants were mainly related to the use of the plant, its local name, habitat, place and time of collection, used parts and categories, fruit and vegetable consumption, way of drug preparation and administration and at last, diseases treated. Plant collection was done during the blooming and fruiting stage, approved by local inhabitants to verify the authenticity of the collected plant. After drying, the specimens were pressed, poisoned and finally mounted on herbarium sheets. Interviews were conducted mostly in fields; otherwise, photographs were shown for identification. Females were interviewed indirectly through male family members. Participants were briefed about the research objectives and were allowed to discontinue the interview at any time. The native language (Urdu and Hindko) has been used as a medium of communication. Later, an English language questionary proforma was filled for each informant.

\section{Identification of plants}

The collected plant species were identified with the available Flora of Pakistan, and their names were identified through the literature search and key plant-related websites such as the plant list (www.theplantlist.org), international plant name index (http://www.ipni.org) and GRIN taxonomy site (http://www.ars-grin.gov/cgi-bin/npgs/html/queries.p 1). For voucher specimens, standard herbarium techniques $(23,24)$ were strictly followed and were deposited in the Hazara University Herbarium, Pakistan for future references.

\section{Quantitative and correlations analysis of ethnomedicinal data}

The obtained data were analyzed and presented using quantitative indices, descriptive statistics and correlation analysis for all the collected plant species.

\section{Relative Frequency Citation (RFC)}

Relative frequency citation was calculated while ignoring the use-categories following the formula (25).

$$
R F C=\frac{F C}{N}(0>F R C>1)
$$

RFC shows the importance of each species in the study area given by the FC (FC is the number of local informants reported the uses of the species) divided by the total number of informants $(\mathrm{N})$.

\section{Use value (UV)}

Use value (UV) determines the importance of each plant species utilized by locals. It was calculated using the following formula (26).

$$
\mathrm{UV}=\Sigma \mathrm{Ui} / \mathrm{N}
$$

Where "UV" indicates the use-value of individual species, "Ui" is the number of uses recoded for a given species by each informant, and " $N$ " represents the total number of informants in the study.

\begin{tabular}{|c|c|c|c|}
\hline Variable & Demographic categories & Number of informants & $\%$ \\
\hline \multirow[t]{2}{*}{ Gender } & Male & 75 & 79 \\
\hline & Female & 20 & 21 \\
\hline \multirow[t]{2}{*}{ Experience } & Traditional healers & 25 & 26 \\
\hline & Local people & 70 & 69 \\
\hline \multirow[t]{3}{*}{ Age groups } & $25-40$ & 13 & 14 \\
\hline & $41-60$ & 19 & 20 \\
\hline & Above 60 & 63 & 66 \\
\hline \multirow[t]{4}{*}{ Education } & Illiterate & 69 & 73 \\
\hline & Primary & 15 & 16 \\
\hline & Middle & 2 & 2 \\
\hline & Matric and above & 9 & 9 \\
\hline
\end{tabular}

Table 1. Demographic information of rural respondents. 


\section{Fidelity level percentage (FL\%)}

Fidelity level (FL) is the percentage of plant specimens that the interviewees claim to be used for the same particular purpose. Fidelity level shows the importance of each species for a specific disease. Its value can be calculated for common diseases as follows:

$$
F L(\%)=\left({ }^{N p} / N\right) \times 100
$$

$N p$ is the number of individuals claiming to utilize a group of plant specimens to cure a specific disease, and $\mathrm{N}$ is the number of people utilizing herbal remedies to treat any illness $(23,27,28)$.

\section{Pearson correlation}

Pearson's correlation, SPSS (ver. 16) tested correlation analysis between the RFC, UV and FL\%.

\section{Results and Discussion}

The study found that 38 species belonging to 37 genera and 27 families are utilized to treat different urinary tract infections (Table 2). The findings on the usage of ethnomedicinal plants for treating urinary tract disorders are displayed alphabetically, along with the botanical/local name, family, habitat, phytoconstituents and type of application. Such traditional medicinal plants have been practiced by the ancient medicinal systems (Ayurveda, Traditional Chinese medicine, Siddha, Unani medicine and Kampo medicine) for a number of diseases (9), like Asparagus racemosus Willd. (root, 5-7 g, powder) used by Siddha for the treatments of urolithiasis $(29,30)$. A large number of preclinical studies support the traditional uses of medicinal plants to prevent kidney stones (31), such as an in vitro experiment (32) found that the aqueous extract of Bryophyllum pinnatum leaves was effective in dissolving calcium phosphate. Another study (29) used the ethanolic extract of Asparagus racemosus on albino rats and found that the concentration of calcium and phosphate was significantly reduced. In short, most of the modern drugs have been originated from the traditional knowledge of medicinal plants by identifying/isolating/modifying the specific chemical constituents for specific diseases and then performing its clinical trials to evaluate their safety and efficacy and also the effective dosage and its route of administration.

\section{Species richness for the treatment of urolithiasis}

A total of 38 species from 37 genera belonging to 27 families were documented, which were used by local

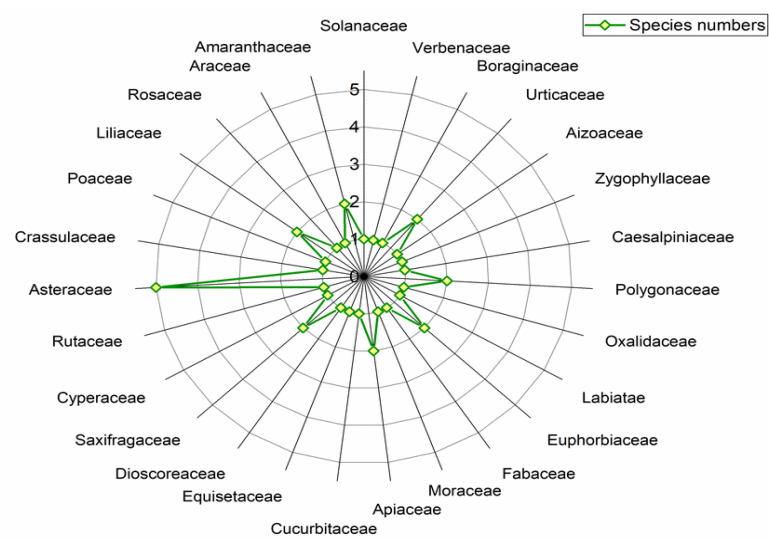

Fig. 2. Taxonomic diversity of medicinal plants used for urolithiasis treatment in Abbottabad District. people for the treatment of urolithiasis in the study area. Asteraceae family (5 species) was the most frequently mentioned among plant families, followed by Amaranthaceae, Liliaceae, Saxifragaceae, Apiaceae, Euphorbiaceae, Polygonaceae and Urticaceae (2 species each) and the other plant families were recorded with only one medicinal plant species each (Fig. 2). Most of the registered plant species in the study area have been practiced by the Ayurveda Pharmacopoeia of India, TCM, Siddha, Unani for the urolithiasis treatment $(2,9)$; some of them have been tested in preclinical studies and some of the claims were scientifically proved for the same diseases as in the therapy/practice.

\section{Ethnomedicinal utilization of plant parts for the treatment of urolithiasis}

A total of eight different plant parts were used for the management of urolithiasis by the local community (Fig. 3). Leaves were the most widely used part $(28.94 \%)$, followed by the whole plant

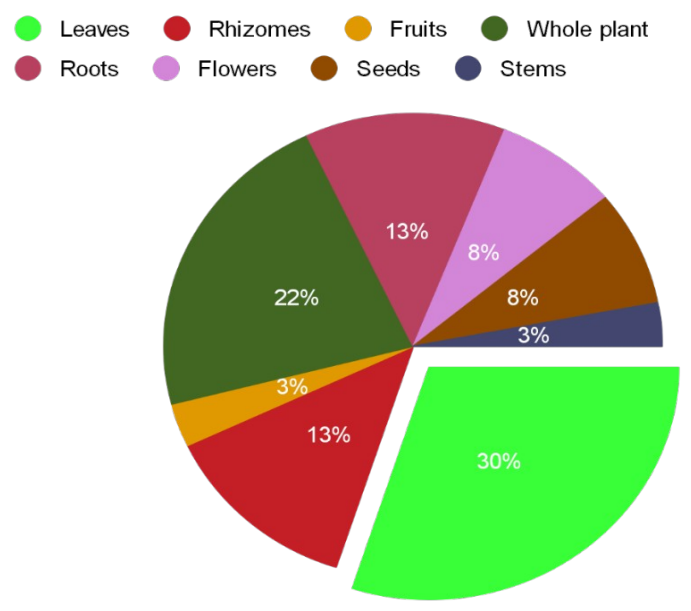

Fig. 3. Ethnomedicinal utilization of plant parts for the treatment of urolithiasis.

(21.05\%), rhizomes and roots (13.5\%), flowers and seeds $(7.89 \%)$ and fruits and stem (2.63\%). Because of the lower level of communication means, ignorance, poverty and the absence of modern therapeutic facilities, the people of the rural areas still depend on traditional medicines for curing various ailments (Table 1). Plant parts and the way of their utilization have a significant role in preventing any disease. It has been found that some of the traditionally used plant parts contain phytoconstituents which can be used as antiurolithiasis agents, such as Bergenia ciliata leaves (ethyl acetate extracts) (33) and Bryophyllum pinnatum leaves extracts (32). In a study, the pulp extracts of Citrullus lanatus were found to be effective against urolithiasis, while the seed extracts did not show any effectiveness (34). In contrast, the seeds of Citrullus lanatus were used traditionally against urolithiasis. However, in this study, most of the reported species based on the part of utilization and their purpose against urolithiasis matched with the phytoconstituents used in pre-clinical stages. Some species were found with no pharmacognostic study records, therefore, recommended for further screening. 
Table 2. Ethnomedicinal plants used for the treatment of urolithiasis.

\begin{tabular}{|c|c|c|c|c|c|c|c|c|c|c|}
\hline \multirow{2}{*}{\multicolumn{2}{|c|}{$\begin{array}{l}\text { Botanical Name, family, Local name } \\
\text { voucher number }\end{array}$}} & \multirow{2}{*}{$\begin{array}{l}\text { Plant parts } \\
\text { used }\end{array}$} & \multirow{2}{*}{$\begin{array}{l}\text { Type of } \\
\text { preparation }\end{array}$} & \multirow{2}{*}{ Disease Treated } & \multirow{2}{*}{$\begin{array}{l}\text { Route and } \\
\text { Dosage }\end{array}$} & \multicolumn{5}{|c|}{ Quantitative Indices } \\
\hline & & & & & & FC & RFC & $\sum \mathbf{U i}$ & UV & FL\% \\
\hline $\begin{array}{l}\text { Achyranthes aspera L. } \\
\text { (Amaranthaceae), HUP- } \\
0002\end{array}$ & Puthkanda & Leaves & Decoction & $\begin{array}{l}\text { Treatment of } \\
\text { calculi }\end{array}$ & $\begin{array}{l}\text { Orally, twice } \\
\text { daily for } 7-10 \\
\text { days }\end{array}$ & 40 & 0.421 & 70 & 0.73 & 50 \\
\hline $\begin{array}{l}\text { Acorus calamus L. } \\
\text { (Araceae), HUP-0003 }\end{array}$ & Bach & Rhizome & Powder & $\begin{array}{l}\text { Diuretic and } \\
\text { useful for } \\
\text { calculous } \\
\text { affections }\end{array}$ & $\begin{array}{l}3 \mathrm{~g} \text { twice daily } \\
\text { for } 7-12 \text { days }\end{array}$ & 29 & 0.305 & 60 & 0.63 & 25 \\
\hline $\begin{array}{l}\text { Aerva lanata L. } \\
\text { (Amaranthaceae), HUP- } \\
0004\end{array}$ & Not Known & Whole plant & Extract & $\begin{array}{l}\text { Diuretic and } \\
\text { lithotriptic and } \\
\text { is used in } \\
\text { lithiasis }\end{array}$ & $\begin{array}{l}\text { Half of the } \\
\text { teacup twice } \\
\text { daily for one } \\
\text { month }\end{array}$ & 55 & 0.578 & 99 & 1.04 & 30 \\
\hline $\begin{array}{l}\text { Agrimonia eupatoria L. } \\
\text { (Rosaceae), HUP-0001 }\end{array}$ & PeeliBooti & Leaves & Decoction & $\begin{array}{l}\text { Alleviates liver, } \\
\text { bladder and } \\
\text { kidney stones }\end{array}$ & $\begin{array}{l}1 \text { teacup twice } \\
\text { daily for two } \\
\text { weeks }\end{array}$ & 49 & 0.515 & 105 & 1.1 & 60 \\
\hline $\begin{array}{l}\text { Asparagus racemosus } \\
\text { Willd. (Liliaceae), HUP- } \\
0005\end{array}$ & Shahghandal & Leaves & Decoction & $\begin{array}{l}\text { Expel stones } \\
\text { from the urinary } \\
\text { tract }\end{array}$ & $\begin{array}{l}1 \text { teacup twice } \\
\text { daily for } 8-12 \\
\text { days }\end{array}$ & 25 & 0.263 & 45 & 0.47 & 80 \\
\hline $\begin{array}{l}\text { Bergenia ciliataL. } \\
\text { (Saxifragaceae), HUP- } \\
0006\end{array}$ & Butpai & Rhizome & Powder & $\begin{array}{l}\text { Release kidney } \\
\text { stones }\end{array}$ & $\begin{array}{l}10 \mathrm{~g} \text { daily for } \\
\text { one week }\end{array}$ & 36 & 0.378 & 70 & 0.73 & 100 \\
\hline $\begin{array}{l}\text { Bergenia stracheyi Engl. } \\
\text { (Saxifragaceae), HUP- } \\
0028\end{array}$ & Butpai & Rhizome & Powder & $\begin{array}{l}\text { Release kidney } \\
\text { stone }\end{array}$ & $\begin{array}{l}10 \mathrm{~g} \text { daily for } \\
\text { one week }\end{array}$ & 22 & 0.231 & 30 & 0.31 & 100 \\
\hline $\begin{array}{l}\text { Bryophyllum pinnatum } \\
\text { (Lam.) Oken. } \\
\text { (Crassulaceae), HUP-0029 }\end{array}$ & Pthar chat. & Leaves & Extract & $\begin{array}{l}\text { Break the kidney } \\
\text { stone }\end{array}$ & $\begin{array}{l}2 \text { teaspoons } \\
\text { twice daily for } 5 \\
\text { to } 8 \text { days }\end{array}$ & 32 & 0.336 & 65 & 0.68 & 90 \\
\hline $\begin{array}{l}\text { Celtis australisL. } \\
\text { (Urticaceae), HUP-0007 }\end{array}$ & Batkalar & Fruits & Decoction & $\begin{array}{l}\text { Remove stones } \\
\text { from the UT, } \\
\text { with frequent } \\
\text { urination } \\
\end{array}$ & $\begin{array}{l}1 \text { teacup twice } \\
\text { daily for two } \\
\text { weeks }\end{array}$ & 41 & 0.431 & 92 & 0.96 & 70 \\
\hline $\begin{array}{l}\text { Cichorium intybus L. } \\
\text { (Asteraceae), HUP-0008 }\end{array}$ & Kasni & Roots & Decoction & $\begin{array}{l}\text { Control } \\
\text { inflammation/pa } \\
\text { in of kidney and } \\
\text { urinary tract } \\
\end{array}$ & $\begin{array}{l}2 \text { teacup thrice } \\
\text { daily for one } \\
\text { week }\end{array}$ & 36 & 0.378 & 87 & 0.91 & 50 \\
\hline $\begin{array}{l}\text { Citrus sinensis (L.) } \\
\text { Osbeck. (Rutaceae), HUP- } \\
0030\end{array}$ & & Fruits & Juice & $\begin{array}{l}\text { Reduce kidney } \\
\text { stone and } \\
\text { support kidney } \\
\text { functions }\end{array}$ & $\begin{array}{l}40 \mathrm{ml} \text { daily for } \\
\text { two weeks }\end{array}$ & 20 & 0.210 & 45 & 0.47 & 70 \\
\hline $\begin{array}{l}\text { Citrullus lanatus (Thunb.) } \\
\text { Matsumura. } \\
\text { (Cucurbitaceae), HUP- } \\
0031\end{array}$ & Tarbooz & Seeds & Powder & $\begin{array}{l}\text { Control kidney } \\
\text { and urinary } \\
\text { tract pain }\end{array}$ & $\begin{array}{l}3 \mathrm{~g} \text { orally for } 4-8 \\
\text { days }\end{array}$ & 25 & 0.263 & 68 & 0.71 & 90 \\
\hline $\begin{array}{l}\text { Conyza canadensis L. } \\
\text { (Asteraceae), HUP-0009 }\end{array}$ & Not Known & Whole plant & Decoction & $\begin{array}{l}\text { Gravel and } \\
\text { kidney } \\
\text { disorders. }\end{array}$ & $\begin{array}{l}1 \text { teacup per day } \\
\text { for } 9-13 \text { days }\end{array}$ & 35 & 0.368 & 88 & 0.92 & 30 \\
\hline $\begin{array}{l}\text { Cyperus rotundus L. } \\
\text { (Cyperaceae), HUP-0010 }\end{array}$ & Muther & Rhizome & Decoction & Dissolve stones & Taken orally & 19 & 0.2 & 59 & 0.62 & 60 \\
\hline $\begin{array}{l}\text { Dioscorea deltoidea Wall. } \\
\text { (Dioscoreaceae), HUP- } \\
0011\end{array}$ & Karins & Rhizome & Cooked & $\begin{array}{l}\text { Kidney } \\
\text { disorders. }\end{array}$ & Taken orally & 31 & 0.326 & 76 & 0.8 & 70 \\
\hline $\begin{array}{l}\text { Eclipta alba Hassak. } \\
\text { (Asteraceae), HUP-0012 }\end{array}$ & Not Known & Leaves & Juice & $\begin{array}{l}\text { Remove stones } \\
\text { from the } \\
\text { gallbladder }\end{array}$ & $\begin{array}{l}25-50 \mathrm{ml} \text { before } \\
\text { breakfast daily } \\
\text { for one week }\end{array}$ & 45 & 0.473 & 98 & 1.03 & 40 \\
\hline $\begin{array}{l}\text { Equisetum ramosissimum } \\
\text { Desf. (Equisetaceae), } \\
\text { HUP-0013 }\end{array}$ & Not Known & Whole plant & Juice & $\begin{array}{l}\text { Urine burning } \\
\text { and expel kidney } \\
\text { stones. }\end{array}$ & $\begin{array}{l}30-50 \mathrm{ml} \text { every } \\
\text { morning for } 6- \\
10 \text { days }\end{array}$ & 39 & 0.410 & 100 & 1.05 & 90 \\
\hline $\begin{array}{l}\text { Eryngium maritimum L. } \\
\text { (Apiaceae), HUP-0014 }\end{array}$ & Kandiari & Roots & Decoction & $\begin{array}{l}\text { Cystitis and } \\
\text { urethritis and } \\
\text { also alleviate } \\
\text { kidney stones. }\end{array}$ & $\begin{array}{l}1 \text { teacup daily } \\
\text { for two weeks }\end{array}$ & 26 & 0.273 & 62 & 0.65 & 40 \\
\hline $\begin{array}{l}\text { Euphorbia prostrata L. } \\
\text { (Euphorbiaceae), HUP- } \\
0032\end{array}$ & Doadal & Whole plant & Powder & Kidney stones. & $\begin{array}{l}\text { 3-5 g twice daily } \\
\text { for 9-12 days }\end{array}$ & 15 & 0.157 & 33 & 0.34 & 50 \\
\hline $\begin{array}{l}\text { Ficus carica L. } \\
\text { (Moraceae), HUP-0033 }\end{array}$ & Angir & Fruits & Decoction & $\begin{array}{l}\text { Destroy urinary } \\
\text { and gall stones }\end{array}$ & $\begin{array}{l}\text { Half of the } \\
\text { teacup before } \\
\text { breakfast for 5- } \\
10 \text { days }\end{array}$ & 19 & 0.2 & 79 & 0.83 & 70 \\
\hline $\begin{array}{l}\text { Flemingia grahamiana } \\
\text { Wight and Arn. } \\
\text { (Fabaceae), HUP-0034 }\end{array}$ & Kamaila & Leaves & Extract & $\begin{array}{l}\text { Diuretics and } \\
\text { cure painful } \\
\text { urination }\end{array}$ & $\begin{array}{l}\text { Two spoons } \\
\text { daily for half a } \\
\text { month }\end{array}$ & 27 & 0.284 & 82 & 0.86 & 60 \\
\hline $\begin{array}{l}\text { Micromeria biflora } \\
\text { (Buch.-Ham. ex D. Don.) } \\
\text { (Labiatae), HUP-0035 }\end{array}$ & & Whole plant & Decoction & $\begin{array}{l}\text { Remove kidney } \\
\text { stones }\end{array}$ & $\begin{array}{l}1 \text { teacup twice } \\
\text { daily for two } \\
\text { weeks }\end{array}$ & 18 & 0.189 & 49 & 0.51 & 50 \\
\hline
\end{tabular}




\begin{tabular}{|c|c|c|c|c|c|c|c|c|c|}
\hline $\begin{array}{l}\text { Phyllanthus niruri L. } \\
\text { (Euphorbiaceae), HUP-0036 }\end{array}$ & & Whole plant & Extract & Stone breaker & $\begin{array}{l}1 \text { teacup daily } 56 \\
\text { for one month }\end{array}$ & 0.589 & 94 & 0.98 & 90 \\
\hline $\begin{array}{l}\text { Polygonum aviculare L. } \\
\text { (Polygonaceae), HUP-0016 }\end{array}$ & Tarubra. & Leaves & Decoction & $\begin{array}{l}\text { Dissolve and } \\
\text { expel kidney } \\
\text { stones }\end{array}$ & $\begin{array}{l}1 \text { teacup daily } 34 \\
\text { for } 12-17 \text { days }\end{array}$ & 0.357 & 66 & 0.69 & 60 \\
\hline $\begin{array}{l}\text { Rumex acetosa L. } \\
\text { (Polygonaceae), HUP-0017 }\end{array}$ & Khatri & Roots & Powder & $\begin{array}{l}\text { Dissolve kidney } \\
\text { and bladder } \\
\text { stones }\end{array}$ & $\begin{array}{l}\text { 5-8 g daily for } \\
6-10 \text { days }\end{array}$ & 0.631 & 109 & 1.147 & 80 \\
\hline $\begin{array}{l}\text { Polygonatum multiflorum } \\
\text { Allioni. (Liliaceae), HUP- } \\
0018\end{array}$ & Adbis & Roots & Decoction & $\begin{array}{l}\text { Dissolves kidney } \\
\text { stones and } \\
\text { promote kidney } \\
\text { functions }\end{array}$ & $\begin{array}{l}1 \text { teacup daily } 24 \\
\text { for } 5-8 \text { days }\end{array}$ & 0.252 & 42 & 0.442 & 50 \\
\hline $\begin{array}{l}\text { Tamarindus indica L. } \\
\text { (Caesalpiniaceae), HUP- } \\
0019\end{array}$ & Imli & Fruits & Decoction & $\begin{array}{l}\text { Eliminate } \\
\text { calculi/stones }\end{array}$ & $\begin{array}{l}1 \text { teacup twice } \\
\text { daily for } 10 \\
\text { days }\end{array}$ & 0.178 & 39 & 0.410 & 70 \\
\hline $\begin{array}{l}\text { Trachyspermum ammi (L.) } \\
\text { Sprague. (Apiaceae), HUP- } \\
0020\end{array}$ & Ajwain & Seeds & Cooked & $\begin{array}{l}\text { Remove kidney } \\
\text { stones }\end{array}$ & $\begin{array}{l}10 \text { g seeds } \\
\text { soaked in } \\
\text { water } \\
\text { overnight, } 29 \\
\text { taking before } \\
\text { breakfast } \\
\text { daily } \\
\end{array}$ & 0.305 & 78 & 0.821 & 57 \\
\hline $\begin{array}{l}\text { Tribulus terrestris L. } \\
\text { (Zygophyllaceae), HUP- } \\
0021\end{array}$ & Gokhru & Seeds & Powder & $\begin{array}{l}\text { Promotes urine } \\
\text { discharge and } \\
\text { removes painful } \\
\text { urine }\end{array}$ & $\begin{array}{l}4-7 \mathrm{~g} \text { twice } \\
\text { daily for 11-15 } 31 \\
\text { days }\end{array}$ & 0.326 & 93 & 0.97 & 90 \\
\hline $\begin{array}{l}\text { Trianthema portulacastrum } \\
\text { L. (Aizoaceae), HUP-0037 }\end{array}$ & ${ }^{\imath}$ kandiari & Leaves & Decoction & $\begin{array}{l}\text { Diuretic and } \\
\text { break the kidney } \\
\text { and bladder } \\
\text { stones }\end{array}$ & $\begin{array}{l}1 \text { teacup daily } 20 \\
\text { for 5-8 days }\end{array}$ & 0.21 & 75 & 0.78 & 80 \\
\hline $\begin{array}{l}\text { Trichodesma indicum (L.) } \\
\text { R. Br. (Boraginaceae), HUP- } \\
0038\end{array}$ & & Leaves & Extract & Stone breaker & $\begin{array}{l}1 \text { spoon thrice } \\
\text { daily for } 9-1322 \\
\text { days }\end{array}$ & 0.231 & 69 & 0.72 & 60 \\
\hline $\begin{array}{l}\text { Solidago virga-aurea L. } \\
\text { (Asteraceae), HUP-0022 }\end{array}$ & Not Known & Leaves & Decoction & $\begin{array}{l}\text { Promote } \\
\text { urination/flush } \\
\text { out kidney and } \\
\text { bladder stones. }\end{array}$ & $\begin{array}{l}1 \text { teacup } \\
\text { thrice daily } 36 \\
\text { for one week }\end{array}$ & 0.378 & 85 & 0.89 & 23 \\
\hline $\begin{array}{l}\text { Urtica dioca L. } \\
\text { (Urticaceae), HUP-0023 }\end{array}$ & Bichubooti & Leaves & $\begin{array}{l}\text { Decoction, } \\
\text { Cooked }\end{array}$ & $\begin{array}{l}\text { Dissolves } \\
\text { calcium } \\
\text { oxalate/renal } \\
\text { stones }\end{array}$ & $\begin{array}{l}\text { Taken orally/1 } \\
\text { teacup twice } \\
\text { daily for } 15-30 \\
\text { days }\end{array}$ & 0.126 & 46 & 0.48 & 40 \\
\hline $\begin{array}{l}\text { Verbena officinalis L. } \\
\text { (Verbenaceae), HUP-0024 }\end{array}$ & Not Known & Whole plant & Decoction & $\begin{array}{l}\text { Remove kidney } \\
\text { stones/disorders }\end{array}$ & $\begin{array}{l}2 \text { teacup twice } \\
\text { daily for } 7-11 \quad 11 \\
\text { days }\end{array}$ & 0.115 & 38 & 0.4 & 25 \\
\hline $\begin{array}{l}\text { Withania somnifera Dunal. } \\
\text { (Solanaceae), HUP-0025 }\end{array}$ & Askand & Roots & Decoction & $\begin{array}{l}\text { Prevent and } \\
\text { treat kidney } \\
\text { damage }\end{array}$ & $\begin{array}{l}1 \text { teacup daily } \\
\text { for } 7-11 \text { days }\end{array}$ & 0.136 & 67 & 0.70 & 32 \\
\hline $\begin{array}{l}\text { Xanthium strumarium L. } \\
\text { (Asteraceae), HUP-0026 }\end{array}$ & Kandiari & Leaves & Decoction & $\begin{array}{l}\text { Treat bladder } \\
\text { infections }\end{array}$ & $\begin{array}{l}1 \text { teacup twice } \\
\text { daily for two } 40 \\
\text { weeks }\end{array}$ & 0.421 & 92 & 0.96 & 60 \\
\hline
\end{tabular}

FC, Frequency citation; RFC, Relative frequency of citation; $\sum$ Ui, Sum of all uses mentioned by each informant for a species; UV, Use values; FL\%, Fidelity level percentage.

\section{Preparations of herbal remedies}

The local communities used the native diversity of medicinal plants for treating urolithiasis disease by following several administrational ways; decoction was the popular and dominant way of preparation

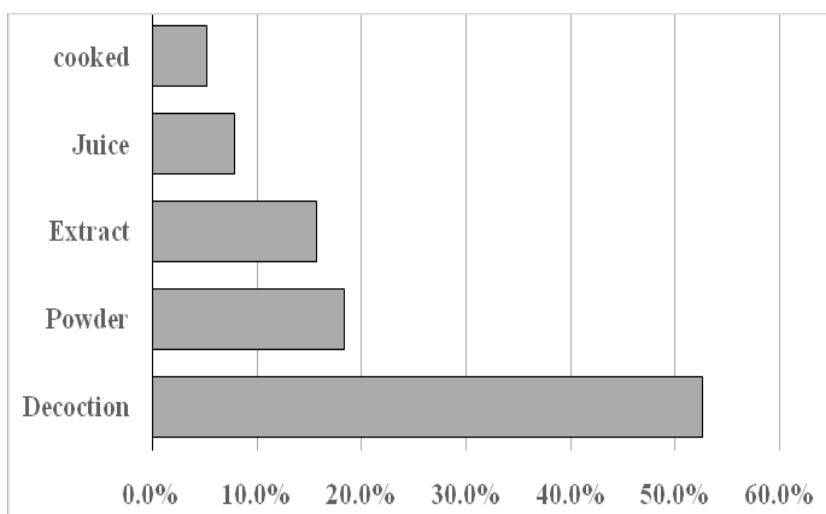

Fig. 4. Types of preparations used against urolithiasis in the study area.
(52.6\%), followed by powder (18.4\%), extracts (15.7\%), juice (7.8\%) and cooked (5.2\%) (Fig. 4). Mostly used medicinal plants demonstrate that approximately 650 species are utilized as mono- or polyherbal remedies and are used in the form of decoction and acts as diuretic substances (31). The preparation of polyherbal remedies is considered less toxic, safe and effective by traditional medicinal systems and is inexpensive than allopathic therapy (2). In traditional therapies, plant parts used, their preparation and the route of administration may have a significant role in the pharmacological action of medicinally used plant taxa.

\section{Important ethnomedicinal species}

The UV of plant species determines the relative importance of plants in the study area. The highest UV was recorded for Rumex acetosa L. (1.14), Agrimonia eupatoria L. (1.1), Equisetum ramosissimum Desf. (1.05), Aerva lanata L. (1.04), Eclipta alba Hassak. (1.03), Phyllanthus niruri L. (0.98), Tribulus terrestris L. (0.97), Xanthium 
strumarium L. and Celtis australis L. (0.96 each), Cichorium intybus L. (0.91), Conyza canadensis L. (0.92), and the other recorded plant species were $<0.90$ use-values. RFC values can be used to identify the most known medicinal plants species among the local peoples. In this study, the high RFC value was recorded for Rumex acetosaL. (0.63), Phyllanthus niruri L. (0.58), Aerva lanata L. (0.57), Agrimonia eupatoria L. (0.51), Eclipta alba Hassak. (0.47), Celtis australis L. (0.43), Achyranthes aspera L. and Xanthium strumarium L. (0.42 each), Equisetum ramosissimum Desf. (0.41), and the other recorded plant species were $<0.4$ RFC value. Use value and RFC value are dynamic as it changes with the area and depends on the local people's traditional knowledge, so the UV and RFC value may vary within the same area or area to area and community to community (35). Correlation analysis between RFC and UV was significant at the 0.01 level $\left(0.803^{* *}\right)$, indicating that the local people have significant traditional knowledge of the cited species (Table 3).

Table 3. Correlation between RFC, UV and FL\% of the recoded medicinal plants

\begin{tabular}{lcccrrrr}
\hline & \multicolumn{3}{c}{ Descriptive } & \multicolumn{3}{c}{ Correlation } \\
\hline Variables & N & Mean & SD & RFC & UV & \multicolumn{1}{c}{ FL\% } \\
\hline RFC & 38 & 0.3147 & 0.13278 & 1 & $0.803^{* *}$ & 0.128 \\
\hline UV & 38 & 0.7471 & 0.24125 & $0.803^{* *}$ & 1 & 0.091 \\
\hline FL & 38 & 61.8947 & 22.28536 & 0.128 & 0.091 & 1 \\
\hline ** represen
\end{tabular}

Fidelity Level percentage shows the importance of each species for specific urolithiasis disease in this study (Table 2). Those species with above $50 \mathrm{FL} \%$ in the study area were considered for further literature search, parts used, pharmacological properties and chemical constituents against urolithiasis. The similarity of traditionally used/reported species was also evaluated against the phytoconstituents assessed in preclinical models of urolithiasis (Table 4). An invitro experiment (33) found that phenolic compounds (ethyl acetate extracts) from the Bergenia ciliata leaves effectively dissolved calcium phosphate and oxalate stones, where cystone was more effective than phenolic compound against urolithiasis. The leaf extracts of Bryophyllum pinnatum and their chemical constituents are useful for preventing kidney stones formation and decreasing urinary calcium levels.

The current reported medicinal plants activities against urolithiasis may be due to the presence of bioactive compounds in the plant parts used traditionally and some of these parts of the plants have been investigated for their bioactive compounds against urolithiasis, while some are still unknown. Previous studies found the effectiveness of the chemical constituents against urolithiasis derived from plant parts that were practiced in the present study, such as Citrullus lanatus and Tribulus terrestris (seed) (34, 36), Equisetum ramosissimum, Oxalis corniculata and Phyllanthus niruri (whole plant) (3739), Zea mays (stem) (40), Asparagus racemosus, Agrimonia eupatoria, Polygonum aviculare, Trichodesma indicum, Xanthium strumarium and Trianthema portulacastrum (leaves) (29, 41-44),
Rumex acetosa (root) (45), Celtis australis, Tamarindus indica, Ficus carica and Citrus sinensis (fruit) (46-48), Dioscorea deltoidea and Cyperus rotundus (rhizome) $(49,50)$. The present study demonstrated that the chemical compounds from plant parts in the modern health systems and their utilization (51) may have been practiced earlier by the ancient traditional medicine systems in their own way and may have been transferred from generation after generation.

Previous studies demonstrated that Bergenia stracheyi had been widely used by traditional Indian, Nepalese and Chinese medicine systems for the treatment of bladder and kidney stones and several other diseases (52-54). Similarly, the present study recorded Bergenia stracheyi with cent fidelity level, used against kidney stones that release stones from the kidney, yet no medical therapy has been performed. Another plant species Flemingia grahamiana was continuously reported in the study area, but it has not been well explored as antiurolithiasis in pre-clinical trials. Therefore, further clinical trials are required, especially of these two species, to record their efficacy against urolithiasis.

\section{Cultural significance}

The WHO promotes and advises the documentation of traditionally used medicinal plants for the treatment of various diseases around the world (55). Various clinical trials have been conducted to evaluate the efficacy and safety of various traditional medicinal plants. About $75 \%$ of drugs currently used directly or indirectly came from traditional medicinal plants (56). Listing of high value ethnomedicinal plants is essential and has a significant value in determining human-plant relationships (57). It is important to involve the local communities in developing useful drugs plants that can socioeconomically uplift them. The present study found that only older people and traditional healers have more knowledge of these useful medicinal plants, which may transfer generation after generation. Hence, proper documentation of ethnomedicinal knowledge of valuable medicinal plants in the country is necessary and should be analyzed using modern research techniques to find useful compounds and molecules, and then should be further evaluated in the preclinical and clinical models for the treatment of various diseases. There is a dire need to find better alternative drugs due to the adverse effects of conventional medicines and herbal medicine can be a possible solution for that purpose only after passing through vigorous chemical and biological screening procedures (58). It is also important that new crops are introduced to ethnobotanists and anthropologists, who can gather and document the traditional knowledge and significance of those plants, which will help to conserve the biodiversity and lead to sustainable utilization of these useful plant resources. The currently collected information will help to drive the research work forward and can help in the scientific evaluation of the safety and efficacy of the traditional herbal remedies used by the tribes, especially the less familiar remedies $(59,60)$. Consequently, people can be informed and guided about the scientifically proven efficacious drug treatments for various diseases, which will improve their health conditions. 
Table 4. Major reported species, phytoconstituents and their pharmacological activities against urolithiasis (evidence from literature)

\begin{tabular}{|c|c|c|c|c|c|}
\hline Botanical Name & Pharmacological Activities & Chemical groups & Constituents/extracts & STU & $\begin{array}{l}\text { Referen } \\
\text { ces }\end{array}$ \\
\hline $\begin{array}{l}\text { Agrimonia } \\
\text { eupatoria L. }\end{array}$ & $\begin{array}{l}\text { Antioxidant, antiasthmatic } \\
\text { diuretic, astringent and gall } \\
\text { bladder disorders }\end{array}$ & $\begin{array}{l}\text { Flavonoids, } \\
\text { terpenoids, tannins, } \\
\text { carbohydrates } \\
\text { glycosides, organic } \\
\text { acids and oils }\end{array}$ & $\begin{array}{l}\text { Ursolic acid, silicic, a-myrin } \\
\text { and thiamine }\end{array}$ & Yes & $(42)$ \\
\hline $\begin{array}{l}\text { Asparagus } \\
\text { racemosus Willd. }\end{array}$ & $\begin{array}{l}\text { Antioxidant, antibacterial and } \\
\text { Diuretic }\end{array}$ & $\begin{array}{l}\text { Flavonoids, terpenoids } \\
\text { and sapogenins }\end{array}$ & $\begin{array}{l}\text { quercetin-3-glucoronide/ } \\
\text { leaves) and ethanolic } \\
\text { extract }\end{array}$ & Yes & $(29,61)$ \\
\hline Bergenia ciliata $\mathrm{L}$. & $\begin{array}{l}\text { Anti-inflammatory, antioxidant, } \\
\text { antibacterial and analgesic }\end{array}$ & $\begin{array}{l}\text { Phenolics and } \\
\text { flavonoid }\end{array}$ & $\begin{array}{l}\text { Alcoholic, butanol, ethyl } \\
\text { acetate and hydro-methanol } \\
\text { extracts }\end{array}$ & Yes & $\begin{array}{l}(33,62, \\
63)\end{array}$ \\
\hline $\begin{array}{l}\text { Bergenia stracheyi } \\
\text { Engl. }\end{array}$ & $\begin{array}{l}\text { antibacterial, antiviral, } \\
\text { antifungal, cytoprotective and } \\
\text { antioxidant }\end{array}$ & $\begin{array}{l}\text { Tannins, flavonoids, } \\
\text { benzanoids, steroids, } \\
\text { coumarins, quinoids } \\
\text { and lactone }\end{array}$ & $\begin{array}{l}\text { Not well explored for } \\
\text { kidney diseases }\end{array}$ & $\begin{array}{l}\text { Not well } \\
\text { explored } \\
\text { for } \\
\text { kidney } \\
\text { diseases }\end{array}$ & $(52,53)$ \\
\hline $\begin{array}{l}\text { Bryophyllum } \\
\text { pinnatum (Lam.) } \\
\text { Oken. }\end{array}$ & $\begin{array}{l}\text { Anti-inflammatory, antioxidant, } \\
\text { antibacterial, analgesics and } \\
\text { nephroprotective }\end{array}$ & $\begin{array}{l}\text { Saponins, tannins, } \\
\text { alkaloids and phenolic } \\
\text { compounds }\end{array}$ & $\begin{array}{l}\text { P.ether, chloroform, } \\
\text { ethanol, acetone and } \\
\text { aqueous extracts }\end{array}$ & Yes & $(32)$ \\
\hline Celtis australis $\mathrm{L}$. & $\begin{array}{l}\text { Antioxidant, cytotoxic, } \\
\text { antibacterial and diuretic }\end{array}$ & $\begin{array}{l}\text { Glycosides, steroids, } \\
\text { saponins, terpenoids, } \\
\text { tannins, alkaloids and } \\
\text { phenolic compounds }\end{array}$ & $\begin{array}{l}\text { Methanol, aqueous and } \\
\text { ethyl acetate extracts }\end{array}$ & Yes & $(48)$ \\
\hline $\begin{array}{l}\text { Citrus sinensis (L.) } \\
\text { Osbeck. }\end{array}$ & $\begin{array}{l}\text { Anti-inflammatory, } \\
\text { antihypertensive, antidiabetic } \\
\text { and diuretic }\end{array}$ & $\begin{array}{l}\text { Phenolics, glycosides, } \\
\text { tannins and saponins }\end{array}$ & $\begin{array}{l}\text { Peel, Ethanolic and aqueous } \\
\text { extracts }\end{array}$ & Yes & $(47)$ \\
\hline $\begin{array}{l}\text { Citrullus lanatus } \\
\text { (Thunb.) } \\
\text { Matsumura }\end{array}$ & $\begin{array}{l}\text { Antioxidant, anti-inflammatory, } \\
\text { antimicrobial, hepatoprotective, } \\
\text { anti-plasmodial, antigiardial } \\
\text { and antiulcerogenic }\end{array}$ & Steroids and alkanes & $\begin{array}{l}\text { Cycloartenyl acetate, } \\
\text { octadecane, Heptacosane } \\
\left(1^{\text {st }}, 2^{\text {nd }}, 3^{\text {rd }} \text { and } 4^{\text {th }} \text { isomers }\right)\end{array}$ & Partial & (34) \\
\hline Cyperus rotundus L. & $\begin{array}{l}\text { Analgesic, antioxidant, anti- } \\
\text { inflammatory, antispasmodic, } \\
\text { antiplatelet, antiallergic, } \\
\text { antimicrobial and wound } \\
\text { healing. }\end{array}$ & $\begin{array}{l}\text { Flavonoids, tannins, } \\
\text { glycosides, alkaloids, } \\
\text { saponins, terpenoids, } \\
\text { oils, carbohydrates } \\
\text { and proteins } \\
\end{array}$ & $\begin{array}{l}\text { Hydroalcoholic extracts, } \\
\text { glycerol, myristic acids, } \\
\text { sitosterols and linoleic acid }\end{array}$ & Partial & $(49,64)$ \\
\hline $\begin{array}{l}\text { Dioscorea deltoidea } \\
\text { Wall. }\end{array}$ & $\begin{array}{l}\text { Antioxidant, contraceptive, } \\
\text { antiarthritic,anti-rheumatic, } \\
\text { anti-constipation, antimicrobial } \\
\text { and diuretic }\end{array}$ & $\begin{array}{l}\text { Phenolics, alkaloids } \\
\text { and saponins }\end{array}$ & Aqueous extracts & Partial & $(50)$ \\
\hline $\begin{array}{l}\text { Equisetum } \\
\text { ramosissimum Desf. }\end{array}$ & $\begin{array}{l}\text { Antibacterial, antiseptic and } \\
\text { astringent, antiurolithiatic, } \\
\text { analgesic, antidiabetic, diuretic } \\
\text { and anti-hemorrhagic }\end{array}$ & $\begin{array}{l}\text { Flavonoids and } \\
\text { alkaloids }\end{array}$ & $\begin{array}{l}\text { Quercetin3-O-glucoside, } \\
\text { kaempferol 3-O-glycoside, } \\
\text { apigenin and 5-O-glucoside }\end{array}$ & Partial & $(38)$ \\
\hline Ficus carica $\mathrm{L}$. & $\begin{array}{l}\text { Anti-inflammatory,litholytic, } \\
\text { astringent, antioxidant, } \\
\text { demulcent and diuretic }\end{array}$ & $\begin{array}{l}\text { Polyphenols, } \\
\text { carbohydrates, } \\
\text { minerals and vitamins }\end{array}$ & $\begin{array}{l}\text { Gallic acid, chlorogenic acid, } \\
\text { syringic acid, }(+) \text { - catechin } \\
\text { and methanolic extracts }\end{array}$ & Yes & $(46)$ \\
\hline $\begin{array}{l}\text { Flemingia } \\
\text { grahamiana Wight } \\
\text { \& Arn. }\end{array}$ & $\begin{array}{l}\text { Antioxidant, antimicrobial, anti- } \\
\text { inflammatory, cytotoxic and } \\
\text { antifungal }\end{array}$ & $\begin{array}{l}\text { Alkaloids, terpenoids, } \\
\text { quinones flavonoids, } \\
\text { coumarins and } \\
\text { phenols }\end{array}$ & $\begin{array}{l}\text { Methanol and ethanolic } \\
\text { extracts }\end{array}$ & $\begin{array}{l}\text { Not well } \\
\text { explored } \\
\text { for } \\
\text { kidney } \\
\text { diseases } \\
\end{array}$ & $(65)$ \\
\hline Oxalis corniculata $\mathrm{L}$. & $\begin{array}{l}\text { Antibacterial, anthelmintic, } \\
\text { astringent, diuretic, } \\
\text { antiarthritis, antiphlogistic and } \\
\text { lithontripic }\end{array}$ & $\begin{array}{l}\text { Phytosterols, tannins, } \\
\text { flavonoids and } \\
\text { glycosides }\end{array}$ & $\begin{array}{l}\text { B-sitosterol, ethyl gallate, } \\
\text { betulin, Oxalic acid and } \\
\text { vitamin C }\end{array}$ & Yes & $(66,67)$ \\
\hline Phyllanthus niruri L. & $\begin{array}{l}\text { Diuretic, anti-inflammatory, } \\
\text { antioxidant, antibacterial, } \\
\text { analgesics and spasmolytic }\end{array}$ & $\begin{array}{l}\text { Tannins, lignans, } \\
\text { glycosides, flavonoids, } \\
\text { alkaloids and } \\
\text { phenylpropanoids }\end{array}$ & $\begin{array}{l}\text { The aqueous extract of the } \\
\text { whole plant and } \\
\text { ellagitannins. }\end{array}$ & Yes & (39) \\
\hline $\begin{array}{l}\text { Polygonum } \\
\text { aviculare } \mathrm{L} .\end{array}$ & $\begin{array}{l}\text { Diuretic, antihypertensive, } \\
\text { antibacterial and antioxidant }\end{array}$ & $\begin{array}{l}\text { Phenols, carboxylic } \\
\text { acids and glycosides }\end{array}$ & $\begin{array}{l}\text { Bryophillin A, } \\
\text { hydroxycinnamic acid, } \\
\text { bersaldegenin-3-acetate and } \\
\text { bryophillin C }\end{array}$ & Yes & $(43,68)$ \\
\hline Rumex acetosa $\mathrm{L}$. & $\begin{array}{l}\text { Anti-inflammatory, } \\
\text { antihypertensive, antidiabetic } \\
\text { and diuretic }\end{array}$ & $\begin{array}{l}\text { Polyphenols, } \\
\text { antraquinones and } \\
\text { tannins }\end{array}$ & $\begin{array}{l}\text { Emodin, palmatin, and } \\
\text { sennosides A and B }\end{array}$ & Yes & $(45)$ \\
\hline $\begin{array}{l}\text { Tamarindus indica } \\
\text { L. }\end{array}$ & Antibiotic and antilithiatic & $\begin{array}{l}\text { Alkaloids, tannins, } \\
\text { flavonoids, terpenoids } \\
\text { and steroids }\end{array}$ & $\begin{array}{l}\text { Caffeic acid, ferulic acid, } \\
\text { chloramphenicol, myricetin } \\
\text { and quercetin. }\end{array}$ & Yes & $(46,69)$ \\
\hline Tribulus terestris L. & $\begin{array}{l}\text { Spasmolytic, diuretic, } \\
\text { analgesics, anti-inflammatory } \\
\text { and antibacterial }\end{array}$ & $\begin{array}{l}\text { Saponin, flavonoids } \\
\text { and alkaloid }\end{array}$ & $\begin{array}{l}\text { n-Butanol, glycolate } \\
\text { oxidase, quercetin. }\end{array}$ & Yes & $(36)$ \\
\hline $\begin{array}{l}\text { Trianthema } \\
\text { portulacastrum } \mathrm{L} .\end{array}$ & $\begin{array}{l}\text { Anti-inflammatory, antioxidant, } \\
\text { antibacterial, analgesic, diuretic } \\
\text { and spasmolytic }\end{array}$ & $\begin{array}{l}\text { Saponins, flavonoids, } \\
\text { tannins, alkaloids and } \\
\text { phenolic compounds }\end{array}$ & $\begin{array}{l}\beta \text {-Cyanin, 5,2'-dihydroxy7- } \\
\text { methoxy-6,8-dimethyl } \\
\text { flavones (Cmethylflavone), } \\
\text { leptorumol, } \beta \text {-sitosterol, } \beta \text { - } \\
\text { glucopyranosides. }\end{array}$ & Yes & $(41,70)$ \\
\hline
\end{tabular}




\begin{tabular}{|c|c|c|c|c|c|}
\hline $\begin{array}{l}\text { Trichodesma } \\
\text { indicum (L.) R. Br. }\end{array}$ & $\begin{array}{l}\text { Anti-inflammatory, Antioxidant, } \\
\text { antimicrobial, antiviral and skin } \\
\text { diseases }\end{array}$ & $\begin{array}{l}\text { Phenols, glycosides, } \\
\text { terpenoids, oils and } \\
\text { tannins }\end{array}$ & $\begin{array}{l}\text { Methanol and } \\
\text { Ethanolicextracts }\end{array}$ & Yes & (71) \\
\hline $\begin{array}{l}\text { Xanthium } \\
\text { strumarium L. }\end{array}$ & $\begin{array}{l}\text { Antiulcerogenic, anti- } \\
\text { proliferative, anti- } \\
\text { inflammatory, analgesic, } \\
\text { antidiabetic and hypoglycaemic, } \\
\text { antiarthritic, diuretic and } \\
\text { renoprotective, antimicrobial, } \\
\text { antihelmintic and anti- } \\
\text { plasmodial. }\end{array}$ & $\begin{array}{l}\text { Terpenoids, steroids, } \\
\text { coumarins, } \\
\text { lignanoids, phenols, } \\
\text { coumarins and } \\
\text { glycosides }\end{array}$ & $\begin{array}{l}\text { Deacetyl xanthumin, } \\
\text { xanthostrumarin and } \\
\text { xanthatin }\end{array}$ & Yes & $\begin{array}{l}(31,44, \\
72)\end{array}$ \\
\hline Zea mays L. & $\begin{array}{l}\text { Anti-inflammatory, antioxidant, } \\
\text { diuretic and nephroprotective }\end{array}$ & $\begin{array}{l}\text { Saponins, flavonoids, } \\
\text { tannins and } \\
\text { alkaloids }\end{array}$ & Corn silk extracts & Yes & $(40,73)$ \\
\hline
\end{tabular}

STU, Similarity with Traditional Utilization

Status of medicinal plants of Abbottabad region

The primary sources of medicinal plants in Pakistan are rangelands and forests, mainly utilized as traditional medicines (74-76). The biodiversity of medicinal plants in the region is under serious threat because of anthropogenic activities, overgrazing, deforestation, timber smuggling, ruthless and unscientific collection (22). These anthropogenic activities lead to the alteration in habitat, fragmentation and severe habitat destruction, which resulted in significant habitat loss. Furthermore, climate change and plant invasions are the other serious threats in this regard $(77,78)$. Both in situ and ex situ conservation measures are required to conserve the regional flora, such as massive reforestation, natural gas supply, community awareness, providing energy efficient cookstoves, and establishing botanical gardens and proper guiding training of medicinal-plant collectors, which may help in flora conservation. However, these efforts will only get results if they are cross-sectoral and promote cooperation and collaboration among the natural resource managers, government agencies, traditional medical practitioners and various other interest groups.

\section{Conclusion}

The current study reported that the local communities widely practice indigenous herbalbased medicines and its utilization remains to be a viable and better alternative therapeutic option for the rural and underprivileged sections of the communities. However, precise traditional knowledge about medicinal plants was found among few people (mostly older people) in the local communities. Therefore, proper exploration, documentation and preservation of traditional ethnomedicinal knowledge are required before it disappears forever and needs to be preserved for future generations. Our survey revealed that there are still some claims that warrant experimental investigations, implying that a lot can still be learned from exploring medicinal plants in the region, which may lead to finding valuable phytoconstituents in plants that are effective against urolithiasis and also other diseases. All these ethnomedicinal plants require a thorough scientific investigation for isolation, identification, biochemical assays, toxicities and evaluation of pharmacological activities of the phytoconstituents, especially of the high-fidelity level plants. This study will help in preserving the useful and immensely important knowledge of traditionally used medicinal plants in the region and promote the importance of ancient healing practices in the coming generations. It also emphasizes the need for a holistic approach to engaging the local communities in the conservation of medicinal plants by sustained harvest and cultivation in their natural habitat due to the immense ecological and pharmacological importance.

\section{Acknowledgements}

The authors thankfully acknowledge the free participation of the traditional healers and other local respondents who provided relevant information about the medicinal plants and made this survey possible.

\section{Authors' contributions}

GMS: Conceptualization, methodology, investigation, formal analysis. NS: Conceptualization, writing, software, statistical analysis, writing and editing. A.N: Conceptualization, critical review and editing. MU, MH, SM and MS: Visualization, data curation. FUK, $\mathrm{NJ}$ and $\mathrm{A}$ : Funding acquisition.

\section{Conflict of interests}

Authors do not have any conflict of interests to declare.

\section{References}

1. Singh A, Chitra V. A Probe on the activity of herbal medicines in nephrolithiasis. Res J Pharm Technol. 2019; 12: 4539-4544. https://doi.org/10.5958/0974-360X.2019.00781.9

2. Kant R, Singh TG, Singh S. Mechanistic approach To herbal formulations used for urolithiasis treatment. Obesity Medicine. 2020: 100266 . https://doi.org/10.1016/ j.obmed.2020.100266

3. Saeed ZI, Hussain SA. Chronic kidney disease in Pakistan: an under-recognized public health problem. Kidney Int. 2012; 81: 1151. https://doi.org/10.1038/ki.2012.47

4. Talati JJ, Hulton S-A, Garrelfs SF, Aziz W, Rao S, et al. Primary hyperoxaluria in populations of Pakistan origin: results from a literature review and two major registries. Urolithiasis. 2018; 46: 187-95. https://doi.org/10.1007/ s00240-017-0996-8 
5. Agawane SB, Gupta VS, Kulkarni MJ, Bhattacharya AK, Koratkar SS, et al. Patho-physiological evaluation of Duranta erecta for the treatment of urolithiasis. J Ayurveda Integr Med. 2019; 10: 4-11. https://doi.org/10.1016/ j.jaim.2017.08.001

6. Nirumand MC, Hajialyani M, Rahimi R, Farzaei MH, Zingue $\mathrm{S}$ et al. Dietary plants for the prevention and management of kidney stones: preclinical and clinical evidence and molecular mechanisms. Int J Mol Sci. 2018; 19: 765. https:// doi.org/10.3390/ijms19030765

7. Bahmani M, Baharvand-Ahmadi B, Tajeddini P, Rafieian-Kopaei M, Naghdi N. Identification of medicinal plants for the treatment of kidney and urinary stones. J Renal Inj Prev. 2016; 5: 129. https://doi.org/10.15171/jrip.2016.27

8. Sundararajan R, Bharampuram A, Koduru R. A review on phytoconstituents for nephroprotective activity. Pharmacophore. 2014; 5: 160-82.

9. Kasote DM, Jagtap SD, Thapa D, Khyade MS, Russell WR. Herbal remedies for urinary stones used in India and China: A review. J Ethnopharmacol. 2017; 203: 55-68. https://doi.org/10.1016/j.jep.2017.03.038

10. Atanasov AG, Waltenberger B, Pferschy-Wenzig E-M, Linder $\mathrm{T}$, Wawrosch C et al. Discovery and resupply of pharmacologically active plant-derived natural products: A review. Biotechnol Adv. 2015; 33: 1582-1614. https://doi.org/ 10.1016/j.biotechadv.2015.08.001

11. Schippmann U, Leaman D, Cunningham A. A comparison of cultivation and wild collection of medicinal and aromatic plants under sustainability aspects. Frontis. 2006: 75-95. https://doi.org/10.1007/1-4020-5449-1_6

12. Shinwari Z. How to sustainably use medicinal plants: looking ahead; In Proceeding of International Workshop held in Islamabad. 2004. pp. 47-56.

13. Chaudhri I. Observations on the medicinal plants of Kaghan Valley. Pak J Forest. 1959; 9: 16-28.

14. Gilani SA, Qureshi RA, Farooq U. Ethnobotanical studies of Ayubia national park district Abbottabad, Pakistan. J Biol Sci. 2001; 1: 284-86. https://doi.org/10.3923/jbs.2001.284.286

15. Ibrar M. Conservation of indigenous medicinal plants and their traditional knowledge found in moist temperate Himalayas Pakistan: Doctoral dissertation, Quaid-i-Azam University Islamabad, Pakistan. 2003.

16. Matin A, Khan MA, Ashraf M, Qureshi RA. Traditional use of herbs, shrubs and trees of Shogran valley, Mansehra, Pakistan. Pak J Biol Sci. 2001; 4: 1101-07. https://doi.org/ 10.3923/pjbs.2001.1101.1107

17. Qureshi RA, Ghufran MA, Gilani SA, Yousaf Z, Abbas G et al. Indigenous medicinal plants used by local women in southern Himalayan regions of Pakistan. Pak J Bot. 2009; 41:1925 .

18. Shah GM, Khan M, Manzoor H, Zafar J. An ethnobotanical note on fuel wood and timber plant species of Siran Valley, Pakistan. J Biol Sci. 2007; 7: 349-53. https://doi.org/10.3923/ jbs.2007.349.353

19. Shah GM, Khan MA. Checklist of Medicinal Plants of Siran Valley, Mansehra, Pakistan. Ethnobotanical leaflets. 2006;2006:6. https://opensiuc.lib.siu.edu/ebl/vol2006/iss1/6

20. Shah GM, Khan MA, Ahmad M, Zafar M, Khan AA. Observations on antifertility and abortifacient herbal drugs. Afr J Biotechnol. 2009;8.

21. Qureshi S, Khan M, Ahmad M. A survey of useful medicinal plants of Abbottabad in northern Pakistan. Trakia J Sci. 2008; 6: 39-51.
22. Majid A, Ahmad H, Saqib Z, Rahman IU, Khan U et al. Exploring threatened traditional knowledge; ethnomedicinal studies of rare endemic flora from Lesser Himalayan region of Pakistan. Rev Bras Farmacogn. 2019; 29: 785-92. https:// doi.org/10.1016/j.bjp.2019.03.005

23. Alexiades MN, Sheldon JW Selected guidelines for ethnobotanical research: a field manual. Advances in Economic Botany, The New York Botanical Garden, Bronx. 1996; 10.

24. Jain SK. Handbook of field and herbarium methods. Today and Tomorrow Printers and Publishers. New Delhi. 1977.

25. Vitalini S, Iriti M, Puricelli C, Ciuchi D, Segale A et al. Traditional knowledge on medicinal and food plants used in Val San Giacomo (Sondrio, Italy)-An alpine ethnobotanical study. J Ethnopharmacol. 2013; 145: 517-29. https://doi.org/ 10.1016/j.jep.2012.11.024

26. Tardío J, Pardo-de-Santayana M. Cultural importance indices: a comparative analysis based on the useful wild plants of Southern Cantabria (Northern Spain). Econ Bot. 2008; 62: 24-39. https://doi.org/10.1007/s12231-007-9004-5

27. Rahman IU, Ijaz F, Afzal A, Iqbal Z, Ali N, et al. Graphical dataset on important medicinal plants used for curing dental issues in Manoor Valley, Mansehra, Pakistan. Data in brief. 2016;9: 1028. https://doi.org/10.1016/j.dib.2016.11.025

28. Rahman IU, Ijaz F, Iqbal Z, Afzal A, Ali N et al. A novel survey of the ethno medicinal knowledge of dental problems in Manoor Valley (Northern Himalaya), Pakistan. J Ethnopharmacol. 2016; 194: 877-94. https://doi.org/10.1016/ j.jep.2016.10.068

29. Jagannath N, Chikkannasetty SS, Govindadas D, Devasankaraiah G. Study of antiurolithiatic activity of Asparagus racemosus on albino rats. Indian J Pharmacol. 2012; 44 : 576. https://doi.org/10.4103/0253-7613.100378

30. Sofia HN, Walter TM. Anti urolithiatic herbs and effective Siddha formulations. World J Pharm Res. 2015;4:892-911.

31. Sruthi S, Majumder S, Kumari S, Kavya TS, Padmaa M. A review on medicinal plants used as diuretics from karnataka state. World J Pharm Res. 2017; 6: 513-36. https://doi.org/ 10.20959/wjpps20178-9791

32. Bansode P, Pawar P, Babar M. In-vitro urolithiatic activity of Bryophyllum pinnatum against experimentally designed calcium oxalate and calcium phosphate stones. British Journal of Pharmaceutical and Medical Research. 2016;01:34-40.

33. Byahatti VV, Pai KV, D’Souza MG. Effect of phenolic compounds from Bergenia ciliata (Haw.) Sternb. leaves on experimental kidney stones. Anc Sci Life. 2010; 30: 14 . PMC3336267

34. Siddiqui WA, Shahzad M, Shabbir A, Ahmad A. Evaluation of anti-urolithiatic and diuretic activities of watermelon (Citrullus lanatus) using in vivo and in vitro experiments. Biomed Pharmacother. 2018; 97: 1212-21. https://doi.org/ 10.1016/j.biopha.2017.10.162

35. Amjad MS, Qaeem MF, Ahmad I, Khan SU, Chaudhari SK et $a l$. Descriptive study of plant resources in the context of the ethnomedicinal relevance of indigenous flora: A case study from Toli Peer National Park, Azad Jammu and Kashmir Pakistan. PLoS One. 2017; 12: e0171896. https://doi.org/ 10.1371/journal.pone.0171896

36. Chhatre S, Nesari T, Kanchan D. Phytopharmacological overview of Tribulus terrestris. Pharmacogn Rev. 2014; 8 : 45. https://doi.org/10.4103/0973-7847.125530

37. Kumar P, Singhal VK. Erratic male meiosis resulting in $2 \mathrm{n}$ pollen grain formation in a $4 x$ Cytotype $(2 n=28)$ of Ranunculus laetus Wall. ex Royle. Sci World J. 2012; 97: 610-1. https://doi.org/10.1100/2012/691545 
38. Albadri HMB. Phytochemical investigation of horsetail (Equisetum arvense L.) grown in Iraq. Ph.D. Thesis, Ministry of Higher Education and Scientific Research, Al-Mustansiriyah University, College of Pharmacy, Baghdad, Iraq, 2016.

39. Kamruzzaman HM, Hoq O. A review on ethnomedicinal, phytochemical and pharmacological properties of Phyllanthus niruri. J Med Plants Stud. 2016;4:173-80.

40. Milind P, Isha D. Zea maize: a modern craze. Int Res J Pharm. 2013; 4: 39-43. https://doi.org/10.7897/22308407.04609

41. Prakash A, Janmeda P, Sharma V. Bioactivity and Pharmacological Potential of Trianthema portulacastrum L. (Angiosperms: Aizoaceae): An Overview. Plant Science Today. 2019; 6: 590-99. https://doi.org/10.14719/pst.2019.6.sp1.678

42. Al-Snafi AE. Arabian medicinal plants with antiurolithiatic and diuretic effects-plant based review (Part 1). IOSR J Pharm. 2018; 8: 67-80.

43. Xu Y, Li M, Liu Z, WANG H-y, JIANG J-y. Research progress in chemical constituents and pharmacological activities of Polygonum aviculare L. J Anhui Agric Univ. 2012; 39: 812-15.

44. Fan W, Fan L, Peng C, Zhang Q, Wang L et al. Traditional uses, botany, phytochemistry, pharmacology, pharmacokinetics and toxicology of Xanthium strumarium L.: A review. Molecules. 2019; 24: 359. https://doi.org/10.3390/molecules 24020359

45. Bello O, Fasinu P, Bello O, Ogbesejana A, Adetunji C et al. Wild vegetable Rumex acetosa L.: Its ethnobotany, pharmacology and phytochemistry-A review. S Afr J Bot. 2019; 125: 149-60. https://doi.org/10.1016/j.sajb.2019.04.018

46. Ahirwar K. Effective healthful medicinal plants as antilithiatic Agents. J Pharmacogn Phytochem. 2019;8:1849-60.

47. Ahmed S, Hasan MM, Mahmood ZA. Antiurolithiatic plants: multidimensional pharmacology. J Pharmacogn Phytochem. 2016;5:4.

48. Badoni R, Kumar Semwal D, Rawat U, Singh Maniyari Rawat M. Chemical constituents from fruits and stem bark of Celtis australis L. Helv Chim Acta. 2011; 94: 464-73. https://doi.org/10.1002/hlca.201000229

49. Jahan N, Bano H, Makbul SAA, Kumar B, Mushir A. Effect of hydroalcoholic extract of Cyperus rotundus L. rhizome against ethylene glycol and ammonium chloride-induced urolithiasis in male sprague-dawley rats. Urological Science. 2019;30:99. https://doi.org/10.4103/UROS.UROS_136_18

50. Khajuria AK, Bisht N. Ethnomedicinal plants used to treat Nephrolithiasis: A case study Pauri (PAURI Garhwal), Uttarakhand. Synthesis. 2016;2:5.

51. Makbul SAA, Jahan N, Kalam MA. Bio-active compounds from Unani medicinal plants and their application in Urolithiasis. Natural Bio-active Compounds: Springer; 2019. pp. 369-407. https://doi.org/10.1007/978-981-13-7205-6_16

52. Hendrychová H, Tůmová L. Bergenia genus-content matters and biological activity. Ceska a Slovenska farmacie: casopis Ceske farmaceuticke spolecnosti a Slovenske farmaceuticke spolecnosti. 2012; 61: 203-09. PMC7730924

53. Kumar P, Javed MS, Tewari A, Bisht K. Phytochemical investigation and in vitro antifungal activity of essential oil from the rhizome of Bergenia stracheyi (Hook. f. \& Thomson) Engl. J Drug Deliv Ther. 2017; 7: 37-43. https://doi.org/ 10.22270/jddt.v7i1.1365

54. Kumar V, Tyagi D. Review on phytochemical, ethnomedical and biological studies of medically useful genus Bergenia. Int J Curr Microbiol App Sci. 2013;2:328-34.

55. Xu W, Zhu L, Dou D, Zu L, Yan S, et al. Formulation and consideration of World Health Organization international clas- sification of traditional medicine. J Tradit Chin Med. 2020;40:157-61. PMID: 32227778

56. Verma S, Singh S. Current and future status of herbal medicines. Veterinary World. 2008;1:347-50. https://doi.org/ 10.5455/vetworld.2008.347-350

57. Nolan JM, Turner NJ. Ethnobotany: The study of peopleplant relationships. Ethnobiology. Hoboken: Wiley-Blackwell. 2011: 133-47. https://doi.org/ 10.1002/9781118015872.ch9

58. Mussin J, Giusiano G Ethno-Phytopharmacology: product validation process based on traditional knowledge of medicinal plants. Agricultural, Forestry and Bioindustry Biotechnology and Biodiscovery: Springer; 2020. pp. 331-53. https://doi.org/10.1007/978-3-030-51358-0_17

59. Sequeira V. Medicinal plants and conservation in São Tomé. Biodivers Conserv. 1994; 3: 910-26. https://doi.org/10.1007/ BF00129666

60. Wanjohi BK, Njenga EW, Sudoi V, Kipkore WK, Moore HL et al. Ethnobotanical study of traditional knowledge, sustainable uses and management of indigenous non-medicinal plants among the Marakwet Community (Embobut Basin), Elgeyo Marakwet County (Kenya). Ethnobot Res Appl. 2020; 20: 1-16. https://doi.org/10.32859/era.20.1.1-16

61. Negi J, Singh P, Joshi G, Rawat M, Bisht V. Chemical constituents of Asparagus. Pharmacogn Rev. 2010;4 (8):215-20. https://doi.org/10.4103/0973-7847.70921

62. Singh A, Bais RT, Singh V. Antimicrobial susceptibility of Myristica fragrans extract against oral pathogens. Int J Curr Microbiol Appl Sci. 2017; 6: 339-43. https://doi.org/ 10.20546/ijcmas.2017.601.041

63. Saha S, Verma RJ. Inhibition of calcium oxalate crystallisation in-vitro by an extract of Bergenia ciliata. Arab J Urol. 2013; 11: 187-92. https://doi.org/10.1016/j.aju.2013.04.001

64. Sivapalan SR. Medicinal uses and pharmacological activities of Cyperus rotundus Linn.-A Review. Int J Sci Res Pub. 2013;3:1-8.

65. Gumula I, Alao JP, Ndiege IO, Sunnerhagen P, Yenesew A et al. Flemingins $\mathrm{G}-\mathrm{O}$, Cytotoxic and Antioxidant Constituents of the Leaves of Flemingia grahamiana. J Nat Prod. 2014; 77: 2060-67. https://doi.org/10.1021/np500418n

66. Kumar A, Rani NS, Sagwal S. An absolute review on Oxalis corniculata Linn. Int J Res Pharm Biomed Sci. 2012;3:117388.

67. Srikanth M, Swetha T, Veeresh B. Phytochemistry and pharmacology of Oxalis corniculata Linn.: A review. Int J Pharm Sci Res. 2012;3:4077.

68. Nikolaeva G, Lavrent'Eva M, Nikolaeva I. Phenolic compounds from several Polygonum species. Chem Nat Compd. 2009; 45: 735. https://doi.org/10.1007/s10600-009-9414-y

69. Goyal B, Alok S, Jain S, Verma A, Kumar M. Phytochemical and pharmacological invastigation on the leaves of Tamarindus indica Linn. For antilithiatic activity. Int J Pharm Sci Res. 2014;5:259.

70. Lakshmi S, Prabhakaran V, Mallikarjuna G, Gowthami A. Antilithiatic activity of Trianthema portulacastrum L. and Gymnema sylvestre R. Br. against ethylene glycol induced urolithiasis. Int J Pharm Sci Rev Res. 2014;25:16-22.

71. Hem K, Sharma V, Kumar D, Singh NK, Gautam DNS. Ethanopharmacology, pharmacology and phytochemistry of Trichodesma indicum (Linn.) R. Br. Indian J Agr Allied Sci. 2015;1:441-50.

72. Panigrahi PN, Dey S, Sahoo M, Choudhary SS, Mahajan S. Alteration in Oxidative/nitrosative imbalance, histochemical expression of osteopontin and antiurolithiatic efficacy 
of Xanthium strumarium L. in ethylene glycol induced urolithiasis. Biomed Pharmacother. 2016;84:1524-32. https:// doi.org/10.1016/j.biopha.2016.11.029

73. Rathod VD, Fitwe P, Sarnaik D, Kshirsagar S. In vitro antiurolithiatic activity of corn silk of Zea mays. Int J Pharm Sci Rev Res. 2013; 21:16-19.

74. Sher H, Ajaz M, Sher H. Sustainable utilization and economic development of some plant resources in Northern Pakistan. Acta Botanica Yunnanica. 2007; 9:207.

75. Ijaz F, Iqbal Z, Rahman I, Ali N, Qadir G et al. The role of plants in human welfare. J Tradit Med Clin Natur. 2017;6:214. https://doi.org/10.4172/2573-4555.1000214

76. Rahman IU, Ijaz F, Afzal A, Iqbal Z, Ali N et al. Contributions to the phytotherapies of digestive disorders: Traditional knowledge and cultural drivers of Manoor Valley, Northern Pakistan. J Ethnopharmacol. 2016; 192: 30-52. https://doi.org/10.1016/j.jep.2016.06.049

77. Shad N, Zhang L, Shah GM, Haifu F, Ilyas M et al. Plant invasion and $\mathrm{N}_{2} \mathrm{O}$ emission in forest ecosystems. Advances in Forest Management under Global Change: IntechOpen; 2020. https://doi.org/10.5772/intechopen.92239
78. Thomas CD, Cameron A, Green RE, Bakkenes M, Beaumont LJ et al. Extinction risk from climate change. Nature. 2004; 427: 145-48. https://doi.org/10.1038/nature02121

\section{Additional information}

Peer review information: Plant Science Today thanks Sectional Editor and the other anonymous reviewers for their contribution to the peer review of this work.

Reprints and permissions information is available at https://horizonepublishing.com/journals/index.php/PST/open_access_policy Publisher's Note: Horizon e-Publishing Group remains neutral with regard to jurisdictional claims in published maps and institutional affiliations.

To cite this article: Shah G M, Shad N, Sajid M, Asma, Naeem A, Khan F U, Jabeen $N$, Urooj $M$, Muhammad $S$, Hasnain $M$. Herbal remedies used for the management of urolithiasis in Abbottabad, Northern Pakistan. Plant Science Today. 2021;8(4):836-847. https://doi.org/10.14719/pst.2021.8.4.1244

Plant Science Today, published by Horizon e-Publishing Group, is covered by Scopus, Web of Science, BIOSIS Previews, Clarivate Analytics, etc. See https://horizonepublishing.com/journals/index.php/PST/indexing_abstracting 